\title{
CAUSAS DE RETRASO EN LA CONSTRUCCIÓN DE PROYECTOS DE AGUA POTABLE Y ALCANTARILLADO EN ECUADOR
}

\section{CAUSES OF DELAY IN THE CONSTRUCTION OF DRINKING WATER AND SEWER PROJECTS IN ECUADOR}

\author{
Fernando Francisco, Castro Carrera ${ }^{1}$; Edwin Patricio, Castro Merino²; Juan Carlos, Osorio López ${ }^{3}$; Juan \\ Enrique, Merizalde Aguirre ${ }^{4}$
}

Recibido 06/08/2021: Aprobado: 01/10/2021

DOI: https://doi.org/10.51372/gacetatecnica231.2

\begin{abstract}
RESUMEN
Es común que los proyectos de infraestructura experimenten retrasos en su ejecución, cuando se producen en la construcción de proyectos de agua potable y alcantarillado se genera demora en el acceso y disponibilidad de servicios básicos de agua y saneamiento para la población, razón por la cual es importante conocer las causas que los producen para así, poder tomar acciones que los reduzcan. Este artículo presenta los resultados de una investigación realizada sobre los retrasos en la construcción de sistemas de agua potable y alcantarillado en Ecuador, las medidas tomadas para corregir tales demoras, las técnicas, herramientas de planificación y control de obras utilizadas. Para el efecto, se realizó una encuesta a base de un cuestionario en el que se requirió la percepción de profesionales que laboran tanto en instituciones públicas como privadas del país, vinculadas a actividades de construcción, elaboración de estudios o fiscalización de proyectos de agua potable y alcantarillado. Se identificó, que las principales causas para los retrasos en estos proyectos lo constituyen la deficiencia en los estudios, las modificaciones técnicas y los incrementos de cantidades de obra, que las ampliaciones de plazo es la medida principalmente aplicada para corregir los retrasos y que el Diagrama de Barras Gantt es la técnica de planificación y control mayormente utilizada.
\end{abstract}

Palabras clave: retrasos en construcción; abastecimiento de agua potable; planificación y control de proyectos; proyectos de agua potable y alcantarillado

\footnotetext{
${ }^{1}$ Fernando Francisco, Castro Carrera. Docente Investigador de la Pontificia Universidad Católica del Ecuador, Facultad de Ingeniería, Ecuador. Correo: ffcastro@ puce.edu.ec ORCID: http://orcid.org/0000-0002-6426-4828 ${ }^{2}$ Edwin Patricio, Castro Merino. Docente Investigador de la Pontificia Universidad Católica del Ecuador, Facultad de Ingeniería, Ecuador.Correo: epcastrom@puce.edu.ec ORCID: https://orcid.org/0000-0002-88452962

${ }_{3}^{3}$ Juan Carlos, Osorio López. Docente Investigador de la Pontificia Universidad Católica del Ecuador, Facultad de Ciencias Naturales y Exactas, Ecuador. Correo: jcosorio@puce.edu.ec ORCID: http://orcid.org/0000-00028829-3623

${ }^{4} J u a n$ Enrique, Merizalde Aguirre. Docente Investigador de la Pontificia Universidad Católica del Ecuador, Facultad de Ingeniería, Ecuador, Correo: jemerizalde@puce.edu.ec y de la Universidad Central del Ecuador, Facultad de Ingeniería y Ciencias Aplicadas, Ecuador, Correo: jemerizalde@uce.edu.ec ORCID: http://orcid.org/0000-0002-8587-373
} 


\section{ABSTRACT}

It is common for infrastructure projects to experience delays in their execution, when they occur in the construction of drinking water and sewerage projects, there is a delay in the access and availability of basic water and sanitation services for the population, which is why It is important to know the causes that produce them in order to take actions to reduce them. This article presents the results of an investigation carried out on the delays in the construction of potable water and sewerage systems in Ecuador, the actions taken to correct such delays, and the techniques, and construction planning and control tools used. For this purpose, a survey was conducted based on a questionnaire that required the perception of professionals who work in both public and private institutions in Ecuador, linked to construction activities, preparation of studies or inspection of drinking water projects and sewerage. It was identified that the main cause for the delays in these projects is the deficiency in the studies, the technical modifications and the increases in work quantities, that the term extensions is the measure mainly applied to correct the delays and that the Diagram Gantt Bar is the most widely used planning and control technique.

Keywords: construction delays; drinking water supply; project planning and control; drinking water and sewerage projects

\section{INTRODUCCIÓN}

Las obras de infraestructura permiten resolver determinado problema o satisfacer alguna necesidad de la población, sin embargo, los retrasos en los plazos, el incremento de costos y una mala calidad de las obras se presentan comúnmente en la construcción de esos proyectos [1]. Cuando se trata específicamente del sector de agua potable y alcantarillado, las demoras en la ejecución afectan a: la oportunidad en que empieza el proyecto en su etapa de funcionamiento, el incremento de costo incide directamente en los niveles tarifarios que se tendrá que aplicar por los servicios prestados, y una deficiente calidad de las obras podría afectar a la calidad del servicio de agua y saneamiento. Por otra parte, dicho incremento implica la asignación de recursos adicionales no previstos, lo que evidentemente afectaría a la disponibilidad de financiamiento para la ejecución de otros proyectos por parte de las entidades promotoras, que en el caso de Ecuador y para los sistemas de agua potable y alcantarillado corresponde a los Gobiernos Autónomos Descentralizados Municipales. Los retrasos e incremento de costos podrían inclusive originar el abandono de las obras, dejándolas inconclusas [2]. De producirse esta situación, en el caso de los sistemas de agua potable y alcantarillado, sería muy perjudicial para los usuarios.

Una buena administración y dirección de una obra permite que éste cumpla sus objetivos y, por el contrario, una dirección ineficaz o la ausencia de ésta pueden producir incumplimiento de plazos, población insatisfecha, entre otros efectos, que terminan afectando a la calidad del servicio y al prestigio del promotor [3]. Para ilustrar esta situación de incumplimiento de plazos, las ricas democracias, tales como Australia, Canadá, Dinamarca, Alemania, Japón y 
Estados Unidos, entre otras, han experimentado incrementos promedio de los cronogramas de ejecución de un $42,7 \%$ y que 7 de cada 10 proyectos han experimentado retrasos [4].

En este contexto y con la finalidad de reducir los incumplimientos de plazo y sus consecuencias, la administración de la construcción tiene entre sus funciones básicas la planeación y el control, que mediante el uso de herramientas de programación determina los objetivos a ser cumplidos en la obra y mide los resultados para implementar correcciones y mejoras en las previsiones realizadas [5]. Actualmente, existen varias técnicas y aplicaciones informáticas disponibles para la planeación de proyectos, no obstante, es frecuente que las obras se retrasen durante su ejecución, por lo que es importante identificar las causas para reducir y, si fuera posible, evitar su incidencia en futuros proyectos.

El objetivo de este artículo es determinar la percepción que tienen los diferentes actores vinculados con la planificación y construcción de sistemas de agua potable y alcantarillado, sobre cuáles son las principales causas para el retraso en la construcción de ese tipo de proyectos en Ecuador, las medidas tomadas para corregir las demoras y las técnicas y herramientas generalmente utilizadas para la planificación y control de proyectos. Para el efecto, se realizó una encuesta a diferentes actores involucrados en los procesos de planificación y construcción de proyectos de agua potable y alcantarillado en el país, lo que permitió recabar información suficiente para cumplir el objetivo planteado.

\section{DESARROLLO}

\subsection{Planeación de Proyectos}

Según [3], para que un proyecto alcance sus objetivos requiere sea implementado varios procesos, entre los que se encuentran los de planificación, ejecución y control, que, entre otras áreas del conocimiento, incluyen la gestión del cronograma, que persigue la terminación del proyecto a tiempo y comprende tanto el desarrollo del mismo como su monitoreo y control. Requiere, además, seleccionar un método de planeación e ingresar la información del proyecto, tal como las actividades, duraciones e interrelaciones entre ellas, en una herramienta de programación. De hecho, existen varios métodos de planificación, desde el tradicional Diagrama de Barras Gantt, pasando por los Diagramas de Redes, hasta el Método del Valor Ganado y las curvas S.

Se dice que un proyecto experimenta un retraso cuando no concluye en el tiempo estipulado, según [6] frecuentemente las obras de construcción experimentan retrasos y para conseguir que cumplan según sus objetivos, se deben seleccionar métodos adecuados de dirección de 
proyectos, cuya utilización empezó a emplearse a inicios del siglo veinte logrando un desarrollo importante hasta la actualidad, sin embargo se reconoce que en el campo de las obras de infraestructura las técnicas habituales podrían no ser eficaces, siendo necesario considerar métodos más efectivos para planificar y controlar los plazos programados. Así, por ejemplo, el método de la cadena crítica [7] o una combinación de las técnicas de la cadena crítica y el valor ganado [6] se han visto como más útiles que las técnicas tradicionales. Así como un cronograma bien realizado es fundamental para el control de un proyecto y para anticipar si éste terminará según los plazos previstos o si sufrirá demoras, su actualización también lo es para reconocer situaciones que derivarían en retrasos [8].

Desafortunadamente, por diferentes causas, que se investigarán en este trabajo, la ejecución de proyectos suele acusar retrasos, con el consecuente incremento de costos y diferimiento de su puesta en marcha. Esta situación, en el caso de proyectos de agua potable y alcantarillado podría llegar a afectar a los usuarios en su calidad de vida, pues podría retrasar su puesta en funcionamiento y/o incrementar los niveles tarifarios con una eventual afectación a la economía familiar.

\subsection{Características Básicas de los Proyectos de Agua Potable y de Alcantarillado}

6 Ya que la investigación se realizó sobre las causas de retraso en la construcción de proyectos de agua potable y alcantarillado, vale recordar las características más relevantes de este tipo de proyectos. Según [9], un sistema de suministro de agua potable es un conjunto de obras cuyo objetivo es satisfacer en cantidad y calidad las necesidades de agua de la población, para cuyo propósito sus componentes básicos son: captación, línea de conducción, planta de potabilización, tanques de reserva y red de distribución; dentro de este último y elemento importante dentro de la red distribución son las conexiones domiciliarias. Es apropiado indicar que en la construcción de estos proyectos se incluye la implementación de un plan de manejo ambiental.

Por otra parte, un sistema de alcantarillado es un conjunto de obras cuyo objetivo es recolectar las aguas residuales que genera la población, así como las aguas de escorrentía, transportarlas y centralizarlas para su tratamiento, antes de descargarlas en un cuerpo hídrico receptor, para cuyo propósito los componentes básicos de que consta son: red de alcantarillado, que incluye colectores, pozos de revisión y conexiones domiciliarias, emisario y planta depuradora de aguas residuales [10]. En este tipo de proyectos también constituye un elemento importante la implementación de un plan de manejo ambiental. Como se indica en [10], el alcantarillado puede ser: unitario, también llamada combinado, si transporta conjuntamente aguas residuales 
y aguas de escorrentía; y, separativo, o separado, si dispone de redes independientes para las aguas residuales y las de escorrentía. Tanto en los sistemas de agua potable como en los de alcantarillado, dependiendo de las condiciones topográficas, podría requerirse de sistemas de bombeo. En Ecuador, los servicios brindados por los sistemas de agua potable y alcantarillado son considerados como servicios públicos básicos [11], es decir, esenciales para la población.

\subsection{Causas de Retrasos en la Construcción}

La mayor parte de los proyectos de infraestructura acusan retrasos durante su construcción debidos a múltiples razones. En este sentido, en [12] se opina que las principales causas en países en desarrollo son las demoras en los pagos al contratista, la disponibilidad financiera del constructor, una programación inadecuada y una gestión incorrecta en sitio. En un sentido similar, respecto de los retrasos en proyectos viales en países en desarrollo, se realizó un ranking de las causas que influyen, determinando, en su orden, las siguientes: demoras en los pagos al contratista, dificultades financieras del promotor, experiencia inadecuada del contratista, escasez de equipo y gestión y supervisión incorrecta en sitio [13]. Sin embargo, en [14] establecieron que si bien es cierto que existen muchas causas que producen retrasos en la construcción, éstos también están directamente relacionados con la cultura organizacional de las empresas constructoras, pues aspectos tales como la toma de decisiones consensuadas y el trabajo en equipo también pueden influir para reducir la incidencia de retrasos.

La magnitud de dichos retrasos en la construcción de los proyectos de infraestructura varía dependiendo de sus características y condiciones particulares. Así, por ejemplo, de un análisis realizado a 90 proyectos uruguayos en su fase de construcción se determinó que en promedio éstos presentaron un retraso de $34,7 \%$ con valores que variaron entre $28,95 \%$ y 40,41\% [15]. En Colombia, los factores que más influyen en las diferencias de tiempo son la planeación, la disponibilidad de maquinaria, las modificaciones a los diseños y las variaciones de la cotización de la moneda [16]. Sin embargo, en Jordania, donde también se investigaron los incrementos de tiempo y costos en la construcción de proyectos públicos de infraestructura, se identificaron 20 razones para las demoras y sobrecostos, de las cuáles las más importantes fueron las condiciones del terreno, el clima y las órdenes de cambio [17].

Así mismo, en Omán las principales causas para los retrasos, relacionadas con el promotor fueron los cambios en el alcance de proyecto, demora en la toma de decisiones y modificaciones a los diseños, y concernientes al contratista fueron la falta de mano de obra y profesionales experimentados, una deficiente administración del contrato y demora en la entrega de materiales [18]. Por otra parte, en Qatar, una investigación realizada respecto de 
los retrasos y sobrecostos en proyectos de construcción pública de vialidad, edificación y drenaje, determinó que la incidencia de retrasos no fue dependiente del tipo, categoría y tamaño de los proyectos, pero sí respecto de la duración de los mismos [19].

Dos actores fundamentales de la construcción son los promotores de los proyectos y los contratistas de las obras, de modo que conocer sus puntos de vista sobre las demoras resulta interesante. Por ejemplo, según los promotores de los proyectos, las tres principales causas para los retrasos de la construcción en Polonia, en orden de importancia, son: errores en los diseños, calidad de mano de obra y condiciones climáticas, y también opinan que, en la construcción, aun cuando se tenga una buena planificación, existe riego de que se presenten retrasos [20]. En cambio, en opinión de los contratistas en un estudio enfocado al distrito de Peshawar, en Pakistán, los factores que más inciden en las demoras, relacionados con los contratistas son los métodos inadecuados de construcción, cambios de subcontratistas, desacuerdos legales, inadecuada comunicación y personal profesional deficiente, mientras que aquellos relacionados con los promotores tienen un mayor efecto en las demoras y corresponden principalmente a las órdenes de cambio, revisiones de la planeación, comunicación deficiente y demora en la toma de decisiones [21].

Por otra parte, en otra investigación se reportó la influencia del tipo de proyecto, la localización y el área, que no suelen ser considerados cuando se analizan las causas de retrasos, y se determinó que efectivamente el tipo de proyecto y su localización sí influyen en las demoras, y que, en cuanto al primer factor analizado, cuando se trata de infraestructura la principal causa es el retraso en los pagos al contratista, mientras que, en relación al segundo factor, generalmente las causas son comunes por región y que en la mayoría de regiones que investigaron, los principales motivos son los retrasos en los pagos y modificaciones en los diseños [22]. Por último, para resumir, vale señalar que según las investigaciones referidas, los retrasos en la construcción de proyectos de infraestructura es un problema generalizado y que sus causas son muy diversas y están relacionadas con el promotor, con el contratista, con los diseños, con la ejecución misma de las obras y con factores externos.

\section{METODOLOGÍA}

La investigación es de tipo cuantitativa, empleándose la técnica de la encuesta para recabar datos de diversos actores relacionados al área de la construcción. Se llevó a cabo en dos fases: la primera, que corresponde a una prueba piloto, dirigida a una muestra de 12 encuestados con el objeto de verificar las bondades del cuestionario y el alcance del procesamiento de datos; y, una segunda fase, de una población inicial conformada por 384 personas, se obtuvo una 
muestra final de 290 que habían participado en al menos una de las fases del ciclo de vida de proyectos de agua potable y/o de alcantarillado en los 5 años anteriores. El personal encuestado, en el caso de pertenecer a una institución, fue seleccionado por las autoridades nominadoras, o por las jefaturas respectivas. Un $63 \%$ de encuestados localizados en la provincia de Pichincha, en la que se localiza la ciudad de Quito, capital de la República de Ecuador, que concentra de modo significativo a entidades públicas, tales como organismos del Gobierno Central, y a entidades privadas, tales como empresas constructoras y consultoras. Un 88,3\% de encuestados ubicados en ciudades de la región Interandina, principalmente en las ciudades de Quito, Ibarra, Tulcán, Riobamba y Ambato), en un 5,9\% de la región Costa, como Portoviejo, Esmeraldas, Santo Domingo de los Colorados y Guayaquil, en un 5,5\% en la región Oriental como las ciudades de Tena y Francisco de Orellana, y en un $0,3 \%$ en la región insular Galápagos.

El objetivo de la encuesta fue obtener información relacionada con las causas para el retraso en la construcción de proyectos de agua potable y alcantarillado, las acciones tomadas para corregir las demoras, y las técnicas y herramientas utilizadas para realizar la planeación y el control de proyectos. El cuestionario como instrumento empleado fue anónimo y se estructuró en tres secciones:

Primera sección: contiene cuatro preguntas relacionadas con información de la institución a la que pertenece el encuestado:

- Tipo de institución

- Actividad que realiza la empresa

- Si pertenece a una entidad pública, selección el tipo

- Sectores en los que interviene la institución.

Segunda sección: contiene cuatro preguntas de opción múltiple relacionadas con información del encuestado:

- Nivel de instrucción

- Ámbito de la experiencia

- Actividades que ha desempeñado

- ¿En cuántos proyectos por sector, ya sea de agua potable o alcantarillado ha participado usted en los últimos 5 años?

Tercera sección: incluye nueve preguntas, de las cuales ocho fueron de opción múltiple y una del tipo cuadrícula de casillas de verificación, cuyo objetivo fue determinar la información sobre los retrasos en la construcción de sistemas de agua potable y alcantarillado en Ecuador, 
y las técnicas y herramientas utilizadas en la planificación y control de los proyectos:

De estos proyectos:

- ¿Cuántos se han ejecutado dentro del plazo inicial (contractual)?

- De los proyectos no terminados dentro del plazo inicial, indique las tres causas más importantes que usted considere inciden en el retraso en cada tipo de proyecto

- De los proyectos de alcantarillado no ejecutados dentro del plazo inicial ¿En qué componentes se producen los retrasos?

- De los proyectos de agua potable no ejecutados dentro del plazo inicial ¿En qué componentes se producen los retrasos?

- ¿Qué acciones se tomaron para corregir los retrasos?

- Califique la efectividad de las acciones tomadas

- ¿Cómo realiza la planificación y control de proyectos?

- Programas empleados para para la planificación y control de proyectos

- ¿En qué etapas del proyecto se usaron estas herramientas?

\section{RESULTADOS}

En las Tablas 1 y 2 se muestran el tipo de institución a la que pertenecen los encuestados y la actividad que realizan.

Tabla 1. Entidad a la que pertenecen los encuestados. Fuente: los autores

\begin{tabular}{ccc}
\hline Tipo de institución & Número de encuestados & Porcentaje (\%) \\
\hline Pública & 120 & 41,4 \\
Privada & 169 & 58,3 \\
Mixta & 1 & 0,3 \\
\hline
\end{tabular}

Tabla 2. Actividad que realiza la empresa en la que laboran los encuestados. Fuente: los autores

\begin{tabular}{ccc}
\hline Actividad & Número & Porcentaje (\%) \\
\hline Construcción & 102 & 35,2 \\
Estudios, fiscalización y & 46 & 15,9 \\
construcción & & \\
Fiscalización & 38 & 13,1 \\
Estudios & 32 & 11,0 \\
Estudios y fiscalización & 28 & 9,7 \\
Estudios y construcción & 24 & 8,3 \\
Fiscalización y construcción & 20 & 6,9 \\
\hline
\end{tabular}

El 58,3\% labora en entidades privadas, empresas constructoras o consultoras, constructores o consultores individuales en libre ejercicio profesional; el 41,4\% en instituciones públicas, empresas públicas municipales, Gobiernos Autónomos Descentralizados, Ministerios. El $35,2 \%$ labora en organizaciones exclusivamente vinculadas con la construcción y el 15,9\% en 
empresas cuyas actividades incluyen la realización de estudios, fiscalización de obras y construcción.

En la Tabla 3 se muestra el tipo de institución pública en que laboran los encuestados que pertenecen a ese tipo de entidades. El 36,7\% pertenece a Gobiernos Autónomos Descentralizados Municipales (GADM) y el 26,7\% a Empresas Públicas Municipales. Vale destacar que en Ecuador los Gobiernos Autónomos Descentralizados Municipales y, por delegación de éste, las Empresas Públicas Municipales tienen la competencia de la prestación de los servicios de agua potable y alcantarillado en sus jurisdicciones.

Tabla 3. Tipo de entidad pública a la que pertenecen los encuestados. Fuente: los autores

\begin{tabular}{ccc}
\hline Tipo de institución & Número & Porcentaje (\%) \\
\hline Empresa Pública Municipal & 32 & 26,7 \\
Gobierno Autónomo Descentralizado Municipal & 44 & 36,7 \\
Gobierno Autónomo Descentralizado Provincial & 13 & 10,8 \\
Ministerio & 11 & 9,2 \\
Empresa Pública & 5 & 4,2 \\
Otro & 15 & 12,5 \\
\hline
\end{tabular}

En las Tablas 4, 5 y 6 se muestra información de los encuestados. El 73,8\% tiene educación superior y el $24,1 \%$ tiene estudios a nivel de maestría; el $81,4 \%$ tiene experiencia en construcción y el $66,6 \%$ en fiscalización de obras. Del número total de encuestados, 255 personas tienen experiencia en proyectos de agua potable y 262 en proyectos de alcantarillado; de los primeros mencionados, el $69,4 \%$ ha participado entre 1 y 5 proyectos en los últimos 5 años y el resto en más de 5 proyectos, y de los segundos nombrados, el 70,6\% ha participado entre 1 y 5 proyectos en los últimos 5 años y el resto en más de 5 proyectos. De esta información se deduce que las personas encuestadas efectivamente han estado vinculadas con la construcción y otras actividades inherentes a los proyectos de agua potable y alcantarillado.

Tabla 4. Nivel de instrucción de los encuestados. Fuente: los autores

\begin{tabular}{ccc}
\hline Nivel de instrucción & Número & Porcentaje (\%) \\
\hline Bachillerato & 1 & 0,3 \\
Tecnología & 3 & 1,0 \\
Superior & 214 & 73,8 \\
Maestría & 70 & 24,1 \\
Doctorado & 2 & 0,7 \\
\hline
\end{tabular}


Tabla 5. Experiencia de los encuestados. Fuente: los autores

\begin{tabular}{ccc}
\hline Experiencia & Número & Porcentaje (\%) \\
\hline Estudios & 174 & 60,0 \\
Construcción & 236 & 81,4 \\
Fiscalización & 193 & 66,6 \\
Administración de contratos & 126 & 43,4 \\
Supervisión de obras & 168 & 57,9 \\
Otra & 11 & 3,8 \\
\hline
\end{tabular}

Tabla 6. Número de proyectos en que han participado los encuestados. Fuente: los autores

\begin{tabular}{ccccc}
\hline $\begin{array}{c}\text { Número de proyectos } \\
\text { en últimos } 5 \text { años }\end{array}$ & $\begin{array}{c}\text { Número de encuestados con } \\
\text { experiencia en proyectos de agua } \\
\text { potable }\end{array}$ & $\begin{array}{c}\text { Número de encuestados } \\
\text { con experiencia en } \\
\text { proyectos de alcantarillado }\end{array}$ \\
\cline { 2 - 5 } & Número & Porcentaje (\%) & Número & Porcentaje (\%) \\
\hline 1 a 5 & 177 & 69,4 & 185 & 70,6 \\
6 a 10 & 39 & 15,3 & 41 & 15,7 \\
11 o más proyectos & 39 & 15,3 & 36 & 13,7 \\
\hline
\end{tabular}

En la Figura 1 se observa que alrededor del $78 \%$ de los encuestados, reportan que tanto los proyectos de agua potable como los de alcantarillado se ejecutan dentro de los plazos contractuales. Este comportamiento similar en los dos tipos de proyectos posiblemente se deba a que tienen características similares desde el punto de vista constructivo, pues ambos comprenden rubros tales como excavación y relleno de zanjas, instalación de tuberías e instalación de acometidas domiciliarias, y al hecho de que generalmente los mismos contratistas suelen ejecutar los dos tipos de sistemas por sus características constructivas similares.

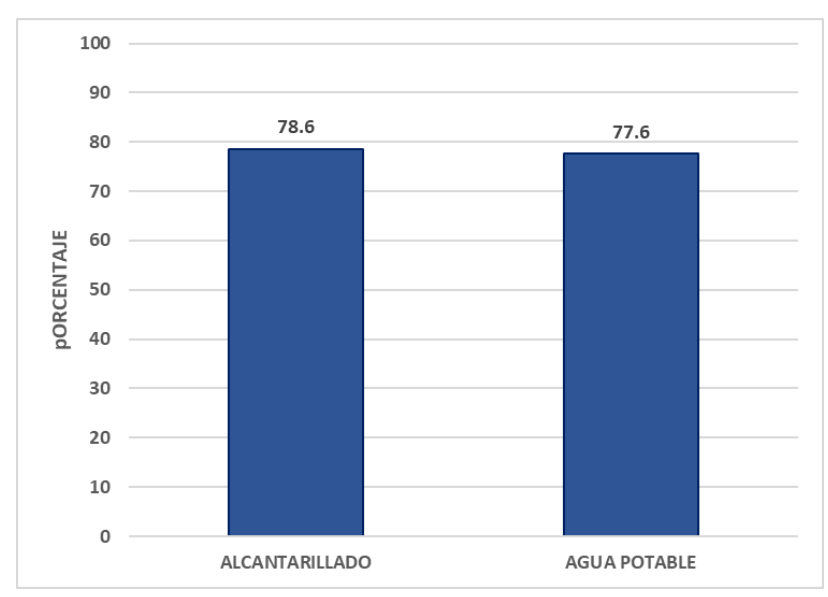

Figura 1. Porcentaje de encuestados que reportan que los proyectos se ejecutan dentro del plazo contractual. Fuente: los autores

En la Tabla 7, para los proyectos no terminados dentro del plazo inicial, se presentan las causas más importantes que los encuestados consideraron inciden en cada tipo de proyecto. De una lista de 13 posibles causas, los encuestados podían escoger las 3 que a su criterio son 
las que más inciden en el retraso; ya que la lista de posibles causas fue lo suficientemente amplia no se previó que pudiese incluirse alguna no contemplada en las opciones disponibles. Entre las causas identificadas, se han establecido como las más importantes, en su orden, la deficiencia en los estudios, modificaciones técnicas en obra e incremento de cantidades de obra respecto de las cantidades inicialmente presupuestadas. Se observó que las causas para los retrasos mantienen la misma tendencia tanto en los proyectos de agua potable como en los de alcantarillado, posiblemente porque desde el punto de vista constructivo tienen una naturaleza similar.

Tabla 7. Causas para el retraso en la construcción de proyectos de agua potable y alcantarillado.

\begin{tabular}{ccccc}
\multicolumn{5}{c}{ Fuente: los autores } \\
\hline Causa & Proyectos de agua potable & \multicolumn{2}{c}{ Proyectos de alcantarillado } \\
\hline & Número & Porcentaje (\%) & Número & Porcentaje (\%) \\
\hline Retraso en pago de planillas & 83 & $11,1 \%$ & 93 & $11,3 \%$ \\
Modificaciones técnicas & 89 & $11,9 \%$ & 97 & $11,8 \%$ \\
Deficiencia en estudios & 101 & $13,5 \%$ & 111 & $13,5 \%$ \\
Condiciones climáticas & 68 & $9,1 \%$ & 81 & $9,8 \%$ \\
Conflictos durante la construcción & 47 & $6,3 \%$ & 49 & $6,0 \%$ \\
Incremento de cantidades de obra & 86 & $11,5 \%$ & 95 & $11,5 \%$ \\
Creación de rubros nuevos & 74 & $9,9 \%$ & 78 & $9,5 \%$ \\
Suscripción de contratos & 50 & $6,7 \%$ & 50 & $6,1 \%$ \\
Trámites burocráticos & 63 & $8,4 \%$ & 67 & $8,1 \%$ \\
Maquinaria insuficiente & 10 & $1,3 \%$ & 13 & $1,6 \%$ \\
Retraso en permisos ambientales & 28 & $3,7 \%$ & 31 & $3,8 \%$ \\
Demora en expropiaciones & 30 & $4,0 \%$ & 34 & $4,1 \%$ \\
Incumplimientos del contratista & 19 & $2,5 \%$ & 25 & $3,0 \%$ \\
\hline
\end{tabular}

En la Tabla 8 se enlistan los componentes de los proyectos de agua potable y alcantarillado en los que se presentan retrasos durante la ejecución. En los primeros, son las conexiones domiciliarias en las que se producen los mayores retrasos, mientras que en los proyectos de alcantarillado las demoras se producen principalmente en las redes de recolección, que comprenden principalmente movimientos de tierras para zanjas y suministro e instalación de tuberías. Estos componentes en los que se producen los mayores retrasos tienen en común que implican actividades en vías urbanas, donde el tráfico vehicular y peatonal suelen interferir con su ejecución.

Las deficiencias en los estudios, que fue identificada como la principal causa para el retraso, podrían generar demoras en la red de recolección de los sistemas de alcantarillado posiblemente porque las características de las zanjas suelen depender de aspectos geotécnicos, que generalmente son soslayados en el diseño de colectores. En el caso de los sistemas de agua potable, los retrasos en las conexiones domiciliarias posiblemente se deban, por un lado, a modificaciones en obra de los diámetros de las acometidas de usuarios especiales que 
requieren especificaciones diferentes a las convencionales y que no fueron definidas en los estudios técnicos del proyecto y, por otro, a demoras constructivas hasta lograr ausencia de fugas en todas las instalaciones.

Tabla 8. Componentes en los que se producen retrasos. Fuente: los autores

\begin{tabular}{cccc}
\hline Construcción de proyectos de agua potable & Construcción de proyectos de alcantarillado \\
\hline Orden & Componente & Orden & Componente \\
\hline 1 & Conexiones domiciliarias & 1 & Red de recolección \\
2 & Línea de conducción & 2 & Obras complementarias \\
3 & Red de distribución & 3 & Conexiones domiciliarias \\
4 & Captación & 4 & Planta depuradora \\
5 & Sistema de bombeo & 5 & Pozos de revisión \\
6 & Planta de potabilización & 6 & Plan de manejo ambiental \\
\hline
\end{tabular}

Los encuestados reportaron las diferentes acciones tomadas durante la construcción para corregir los retrasos; para el efecto, de un listado podían escoger aquellas que se aplicaron en sus proyectos. En la Tabla 9 se muestran tales acciones, se observa que tanto en los proyectos de agua potable como en los de alcantarillado, las tres principales acciones que se tomaron, en su orden, son: ampliaciones de plazo, jornadas adicionales de trabajo y contratación de personal adicional. Vale señalar que las ampliaciones de plazo no corrigen los retrasos propiamente, sino que los legalizan desde un punto de vista contractual.

Tabla 9. Acciones tomadas para corregir retrasos en la ejecución. Fuente: los autores

\begin{tabular}{ccccc}
\hline \multirow{2}{*}{ Acción tomada } & \multicolumn{2}{c}{ Proyectos de agua potable } & \multicolumn{2}{c}{ Proyectos de alcantarillado } \\
\cline { 2 - 5 } & Número & Porcentaje (\%) & Número & Porcentaje (\%) \\
\hline Ninguna & 5 & 1,1 & 10 & 2,0 \\
Ampliación de plazo & 147 & 30,9 & 157 & 31,0 \\
Uso de horas extras & 66 & 13,9 & 75 & 14,8 \\
Contratación de personal adicional & 90 & 18,9 & 93 & 18,4 \\
Reducción de cantidades de obra & 10 & 2,1 & 14 & 2,8 \\
Jornadas adicionales de trabajo & 93 & 19,6 & 98 & 19,4 \\
Subcontratos & 48 & 10,1 & 47 & 9,3 \\
Otras & 16 & 3,4 & 12 & 2,4 \\
\hline
\end{tabular}

Para evaluar la efectividad de las acciones tomadas se pidió que los encuestados las valoren cualitativamente considerando la siguiente escala: excelente, muy buena, buena, regular y mala. En la Figura 2 se puede observar qué a criterio de los encuestados, las ampliaciones de plazos contractuales y la contratación de personal adicional son las que han resultado ser más efectivas, entre buena y excelente, mientras que la reducción de cantidades de obra es la que ha presentado menor efectividad. Evidentemente, el incremento de personal va a incidir directamente en la recuperación de los tiempos perdidos, mientras que la reducción de cantidades de obra podría incidir negativamente en la calidad o cobertura de los proyectos. 


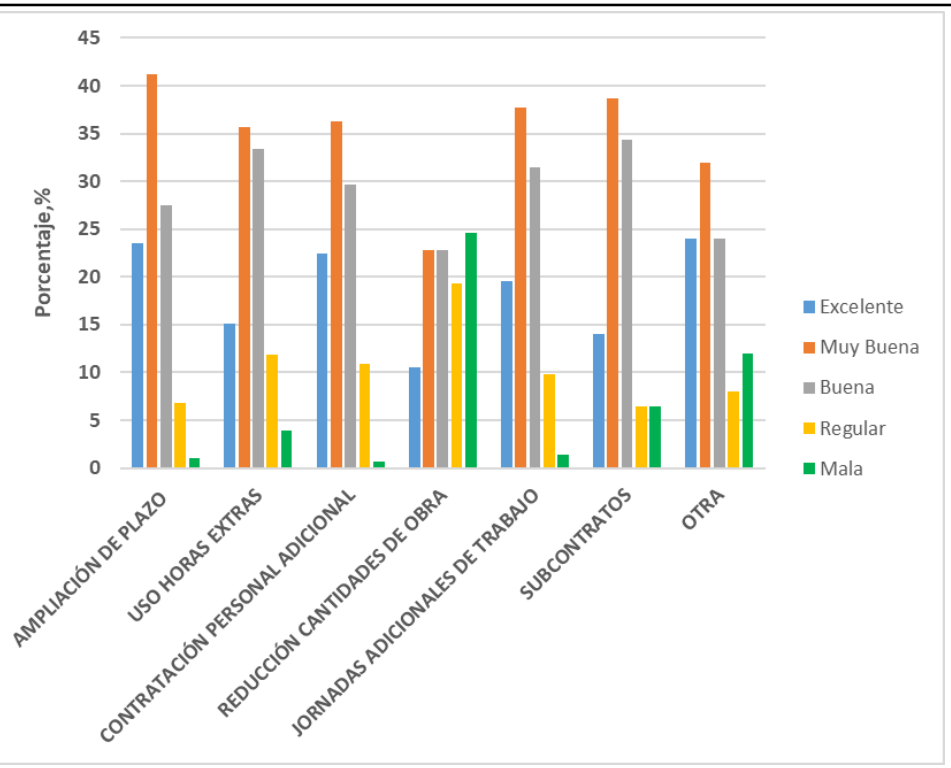

Figura 2. Efectividad de las acciones tomadas. Fuente: los autores

Por otra parte, se requirió que los encuestados informen sobre las técnicas o metodologías de planificación y control de proyectos que utilizan (ver Tabla 10), las herramientas informáticas que han empleado (ver Tabla 11) y el momento o etapa del proyecto en que usaron dichas herramientas (ver Tabla 12). Esto permitió establecer si la forma y oportunidad de realizar la planeación y control podría estar también influyendo en la incidencia de retrasos. En los tres casos, los encuestados podían escoger más de una opción. La metodología mayormente utilizada a criterio de los encuestados es el Diagrama de Barras Gantt, la cual, si bien es una técnica fácil de aplicar, según [23] es una forma de planificación de carácter general que requiere ajustarse en programaciones de corta duración y con mayor especificidad para lograr mayor eficacia y evitar los atrasos por una inadecuada planeación.

Tabla 10. Técnicas utilizadas en la planificación y control de proyectos. Fuente: los autores

\begin{tabular}{ccc}
\hline Técnicas aplicadas & Número & Porcentaje (\%) \\
\hline Diagrama de red & 99 & 24,2 \\
Curvas S & 70 & 17,1 \\
Diagrama de Barras Gantt & 191 & 46,7 \\
Línea de Balance & 22 & 5,4 \\
Otra & 5 & 1,2 \\
Ninguna & 22 & 5,4 \\
\hline
\end{tabular}

Tabla 11. Programas informáticos utilizados en la planificación y control de proyectos. Fuente: los autores

\begin{tabular}{ccc}
\hline Programa informático & Número & Porcentaje (\%) \\
\hline MS Project & 215 & 42,8 \\
Primavera & 12 & 2,4 \\
MS Excel & 160 & 31,9 \\
Proexcel & 96 & 19,1 \\
Otro & 4 & 0,8 \\
Ninguno & 15 & 3,0
\end{tabular}


Tabla 12. Momento en que se usaron las herramientas de planificación y control de proyectos. Fuente: los autores

\begin{tabular}{ccc}
\hline Momento o etapa del proyecto & Número & Porcentaje (\%) \\
\hline Etapa de estudios & 190 & 30,0 \\
Inicio de la obra & 136 & 21,5 \\
Durante la ejecución de la obra & 218 & 34,4 \\
Finalización de la obra & 74 & 11,7 \\
Ninguna & 15 & 2,4 \\
\hline
\end{tabular}

Por otra parte, el programa informático mayormente usado es Microsoft Project, que efectivamente es una herramienta ampliamente utilizada en planeación de proyectos; sin embargo, se observa que la segunda herramienta utilizada es MS Excel, que no es precisamente un software especializado en este tema. Finalmente, respecto de la etapa del proyecto en la que se usan las herramientas de planificación, los encuestados se pronunciaron porque su utilización se circunscribe principalmente a la de ejecución de las obras más que a la de estudios, lo cual posiblemente también incidió en el desfase que se evidencia en los avances reales y los planificados, ya que el estudio de tiempos del proyecto debería iniciarse prioritariamente en la fase de consultoría, durante la realización de los estudios técnicos del proyecto, para definir de mejor manera los plazos contractuales referenciales y, por ende, la planificación inicial que realiza el contratista, lo que contribuiría a reducir las distorsiones entre la planificación y los avances reales.

De lo anterior, podría inferirse que la técnica que mayormente se utiliza en la planeación y control, el uso de herramientas que no son específicas para esa actividad y el momento en que generalmente se las utiliza, no ayudan a lograr un mejor ajuste entre los avances planificados respecto de los reales, favoreciendo la incidencia de retrasos.

\section{CONCLUSIONES}

Un porcentaje importante de sistemas de agua potable y alcantarillado que se ejecutan en Ecuador acusan retrasos durante su ejecución, volviéndose crítica esta situación en este tipo de proyectos, en los que las demoras retrasan la puesta en marcha de servicios básicos para la población, por lo que se vuelve imperativo que éstos concluyan a tiempo. El estudio reflejó que los retrasos en la construcción de este tipo de proyectos en Ecuador se deben principalmente a deficiencias en los estudios, modificaciones técnicas e incrementos de cantidades de obra durante la construcción, factores que suelen ser recurrentes en la ejecución de la mayor parte de obras de infraestructura. Por otra parte, se determinó que la medida que principalmente se adopta para corregir los retrasos es la ampliación de plazo. Desde el punto de vista de las técnicas mayormente utilizadas en la planeación de proyectos, el estudio 
identificó al Diagrama de Barras Gantt como la técnica principalmente utilizada.

Se recomienda, por una parte, la realización de nuevos estudios que aborden aspectos específicos sobre qué tipo de modificaciones técnicas se realizan durante la construcción y cuáles son las deficiencias técnicas encontradas en los estudios y diseños de los proyectos de agua potable y alcantarillado que causan retraso en su construcción, y por otra, ampliar la investigación con encuestados de ciudades que en el presente estudio tuvieron una baja participación.

\section{AGRADECIMIENTOS}

Los autores expresan su agradecimiento a la Pontificia Universidad Católica del Ecuador que, por medio de la Dirección de Investigación, financió el proyecto “Elaboración de curvas 'S' patrón, para la planeación de proyectos de construcción en Ecuador, según su naturaleza", del cual es parte el presente estudio. Se reconoce también a los ahora ingenieros civiles Katherine Vizuete, Karina Granja y Steven Rueda por su participación en la realización de las encuestas.

\section{REFERENCIAS}

[1] J. K. Larsen, G. Q. Shen, S. M. Lindhard y T. D. Brunoe, "Factors Affecting Schedule Delay, Cost Overrun, and Quality Level in Public Construction Projects" ASCE Journal of Management in Engineering, vol. 32, no. 1, 2016, doi: 10.1061/(ASCE)ME.19435479.0000391

[2] N. Rudeli, E. Viles, J. González y A. Santilli, "Causas de Retrasos en Proyectos de Construcción: Un análisis cualitativo" Memoria Investigaciones en Ingeniería, no. 16, pp. 71-84, 2018

[3] Project Management Institute, A Guide to the Project Management Body of Knowledge (Guía de los fundamentos para la dirección de proyectos. Guía del PMBOK), 6a ed., Pennsylvania, 2017

[4] A. Ansar, B. Flyvbjerg, A. Budzier y D. Lunn "Does infrastructure investment lead to economic growth or economic fragility? Evidence from China" Oxford Review of Economic Policy, vol. 32, no. 3, pp. 360-390, 2016. doi:10.1093/oxrep/grw022

[5] S. Arboleda y E. Serna, Presupuesto y programación de obras conceptos básicos, 2a ed, Medellín: Fondo Editorial ITM, 1982

[6] E. Vázquez, Aplicación del método de la cadena crítica al sector de la construcción, Tesis de Doctorado, Universidad de Santiago de Compostela, Lugo, 2018. Recuperado de https://www.educacion.gob.es/teseo/imprimirFicheroTesis.do?idFichero=YiTNm7Kw47 $8 \% 3 \mathrm{D}$

[7] K. Araszkiewicz, "Application of Critical Chain Management in Construction Projects Schedules in a Multi-Project Environment: a Case Study" Procedia Engineering, vol. 182, 
pp. 33-41, 2017. doi: 10.1016/j.proeng.2017.03.108

[8] S. Sepasgozar, R. Karimi, S. Shirowzhan, M. Mojtahedi, S. Ebrahimzadeh y D. McCarthy, "Delay Causes and Emerging Digital Tools: A Novel Model of Delay Analysis, Including Integrated Project Delivery and PMBOK” Buildings, 9, 191, 2019 doi:10.3390/buildings9090191

[9] A. Trapote, Infraestructuras Hidráulico-Sanitarias I. Abastecimiento y distribución de agua, 2a ed., Alicante: Publicaciones Universidad de Alicante, 2013.

[10] A. Trapote, Infraestructuras Hidráulico-Sanitarias II. Saneamiento y drenaje urbano, 2a ed. Alicante: Publicaciones Universidad de Alicante, 2013.

[11] Asamblea Nacional de la República del Ecuador, Ley Orgánica de Recursos Hídricos, Usos y Aprovechamiento del Agua, Registro Oficial No. 305, segundo suplemento, Quito, 2014.

[12] M. Islam y B. Trigunarsyah, "Construction Delays in Developing Countries: A Review" KICEM Journal of Construction Engineering and Project Management, vol. 7, no. 1, pp.1-12, 2017. https://doi.org/10.6106/JCEPM.2017.3.30.001

[13] G. Mejía, O. Sánchez, K. Castañeda y E. Pellicer, "Delay causes in road infrastructure projects in developing countries" Revista de la Construcción, vol. 19, no. 2, pp. 220-234, 2020. https://doi.org/10.7764/rdlc.19.2.220-234

[14] D. Arditi, S. Nayak y A. Damci, "Effect of organizational culture on delay in construction" International Journal of Project Management, vol. 35, pp.136-147, 2017. http://dx.doi.org/10.1016/j.ijproman.2016.10.018

[15] N. Rudeli, E. Viles y A. Santilli, “A Construction Management Tool: Determining a Project Schedule Typical Behaviors Using Cluster Analysis” World Academy of Science, Engineering and Technology, International Journal of Civil and Environmental Engineering, vol. 12, no. 5, pp. 485-492, 2018

[16] S. Lozano Sara, I. Patiño, A. Gómez-Cabrera y A. Torres, "Identificación de factores que generan diferencias de tiempo y costos en proyectos de construcción en Colombia" Ingeniería y Ciencia, vol. 14, no. 27, p. 117-151, 2018, doi: 10.17230/ingciencia.14.27.6

[17] N. Al-Hazim, Z. A. Salem y H. Ahmad, "Delay and Cost Overrun in Infrastructure Projects in Jordan" Procedia Engineering, vol. 182, pp. 18-24, 2017. doi: 10.1016/j.proeng.2017.03.105

[18] T. Al Amri y M. Marey-Pérez, "Towards a sustainable construction industry: Delays and cost overrun causes in construction projects of Oman" Journal of Project Management, vol. 5, no. 2, pp. 87-102, 2020. doi: 10.5267/j.jpm.2020.1.001

[19] A. Senouci, A. Ismail y N. Eldin, "Time Delay and Cost Overrun in Qatari Public Construction Projects" Procedia Engineering, vol. 164, pp. 368-375, 2016. doi: 
10.1016/j.proeng.2016.11.632

[20] M. Gluszak y A. Lesniak, "Construction delays in clients opinion - multivariate statistical analysis" Procedia Engineering, vol. 123, pp. 182 - 189, 2015. doi: 10.1016/j.proeng.2015.10.075

[21] Z. K. Bangash, "Analyzing the Causes of Delays in Construction Projects for Peshawar: Contractor Perception" Journal of Emerging Trends in Applied Engineering, Vol. 1, No. 1, pp. 13-18, 2016.

[22] M. Z. Ramli, M. A. Malek, B. Hamid, N. T. Roslin, M. E. M. Roslan, S. Norhisham y M. F. Mohd, "Influence of Project Type, Location and Area towards Construction Delay: a Review on Significance Level of Delay Factors" International Journal of Engineering \& Technology, vol. 7, no. 4.35, pp. 392-399, 2018. DOI: 10.14419 / ijet.v7i4.35.22769

[23] H. Porras, O. Sánchez y J. Galvis, "Filosofía Lean Construction para la gestión de proyectos de construcción: una revisión actual" AVANCES Investigación en Ingeniería, vol. 11, no. 1, pp. 32-53, 2014. 